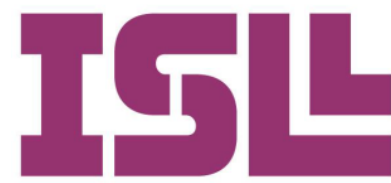

Número 4.

Julio de 2015

\title{
Una clasificación de las inferencias pragmáticas orientada a la didáctica
}

\author{
Juan C. Ripoll Salcedo
}

Colegio Santa María la Real de Sarriguren

Pág. 107 a la 122

\section{Keywords}

Education, inference, reading comprehension, taxonomy

\begin{abstract}
:
Nowadays it is accepted that the ability to make inferences is essential to reading comprehension. Pragmatic inferences are classified according to various criteria, none of these taxonomies is commonly accepted and they have been hardly used in education.

The aim of this paper is to propose an inference taxonomy with the following characteristics: it must be simple, easy to understand and useful for the classroom. Without a

According to a review of the literature on inference making the proposed taxonomy is compatible with previous ones, also with those which are focused on teaching. There are several experimental studies showing that children and adolescents perform the proposed five kinds of inferences when they understand texts. Despite these data, the proposed taxonomy is tentative and should be used with caution because it lacks empirical support.
\end{abstract} suitable taxonomy there is a risk that teachers do not take into account, when assessing or planning classroom activities, all types of inferences that students should use for a good understanding.

The taxonomy was made from the following principle: inferences provide information that does not appear explicitly in the text, so you can ask questions about this information. From this principle it is proposed the existence of five types of inferences that respond to five types of questions. The five main questions are: "what or who is the text alluding to?", "what is the relationship between ... and ...?", "what can be predicted knowing that ...?", "what else can you say about this? "and" what does it all mean? ". 
La palabra inferencia se utiliza en campos como la lógica, la estadística, la lingüística o la psicología. Cada una de estas disciplinas emplea sus propias definiciones del término, pero casi todas ellas tienen en común que hablan de la formación de una conclusión a partir de la información disponible.

Se habla de inferencias deductivas cuando la conclusión a la que se llega es lógica a partir de los datos disponibles. Por ejemplo, si decimos que "Marta es mayor que Jaime y Jaime es mayor que Laura", una conclusión lógica sería que Marta es mayor que Laura. Si, además, las premisas son verdaderas, la conclusión es cierta.

Se suele distinguir otro tipo de inferencias, que son las inductivas o pragmáticas. En este caso la conclusión que se infiere es verosímil, es decir, hay más o menos probabilidades de que sea cierta, pero no se puede garantizar su certeza. El siguiente texto puede servir como ejemplo:

\footnotetext{
Augusto es un ensayo histórico apasionante que se puede leer como una novela. En los idus de marzo del año 44 antes de Cristo, Julio César fue asesinado a los pies de las estatua de Pompeyo. En ese momento Octavio no era más que un oscuro adolescente recién adoptado por el primer hombre de Roma. Ante el magnicidio, dio un paso al frente y se proclamó su legítimo heredero y sucesor. Nadie le tomó en serio, excepto los antiguos soldados de Julio César. En pocos meses formó un ejército y consiguió alzarse como uno de los tres hombres más poderosos del momento, con Marco Antonio y Lépido.
}

Si Octavio, que había sido adoptado por Julio César, se proclamó su legítimo heredero, podemos pensar que Julio César no tuvo descendencia biológica. Ese pensamiento sería una inferencia pragmática. Lo que distingue a estas inferencias pragmáticas de las inferencias lógicas es que las pragmáticas son cancelables. Por ejemplo alguien podría decir que tal vez César sí tuvo descendencia biológica, pero que no fueron hijos reconocidos. De hecho, hay historiadores que consideran que el hijo mayor de Cleopatra, Cesarión, fue hijo de Julio César. Las inferencias lógicas no son cancelables. Si Marta es mayor que Jaime y Jaime es mayor que Laura, no podemos decir que a lo mejor Laura es mayor que Marta, porque las premisas ya excluyen esa posibilidad.

Cuando en el campo de la educación se utiliza la palabra "inferencia", se suele hacer referencia a las inferencias pragmáticas. La palabra "inferencia" aparece actualmente en materiales escolares relacionados con la lengua o la comprensión lectora (por ejemplo, en Fernández, 2008), en tests de evaluación de la habilidad lectora (por ejemplo, Cuetos Rodríguez, Ruano y Arribas, 2007; Abusamra, Ferreres, Raiter, de Beni y Cornoldi, 2010) y, recientemente, en los currículos oficiales (Real Decreto 126/2014).

La preocupación de la escuela por el papel de las inferencias en la comprensión y por el desarrollo de las habilidades inferenciales en el alumnado es algo positivo, ya que en los últimos años se ha puesto de manifiesto la importancia de la construcción de

ISL, vol. 4, 2015, págs. 107-122 ISNN: $2340-8685$
Ripoll Salcedo, J. C. (2015): Una clasificación de las inferencias pragmáticas orientada a la didáctica, Investigaciones Sobre Lectura, 4, 107-122. 
inferencias que ha llegado a ser considerada como "el núcleo de la comprensión e interpretación de la realidad y, por tanto, uno de los pilares de la cognición humana" (León, 2003 p. 23). Además, en el caso concreto de la mejora de la comprensión lectora en español, la construcción de inferencias es una de las estrategias más utilizadas en los métodos que han mostrado mayor eficacia (Ripoll y Aguado, 2014).

\section{La clasificación de las inferencias}

Cuando la preocupación es conseguir que los alumnos sean hábiles en construir las inferencias que necesiten para comprender adecuadamente los textos, es muy importante tener en cuenta que existen distintos tipos de inferencias pragmáticas.

Volviendo al texto sobre Octavio, una representación adecuada de su significado tendría que incluir varias informaciones que no aparecen en el texto. Hay varias referencias como "el primer hombre de Roma" que deben ser vinculadas con el personaje al que se están refiriendo (Julio César). Si se examina el texto con detenimiento, hay una oración que es realmente misteriosa, se trata de "nadie le tomó en serio, excepto los antiguos soldados de Julio César". ¿Por qué no tomaron en serio que Octavio se proclamase heredero de su padre adoptivo cuando era algo esperable y legal? ¿Por qué los antiguos soldados de César sí le tomaron en serio y otros no? En realidad, esa oración no tiene mucho sentido si el lector no aporta algunas informaciones, como que proclamarse sucesor de Julio César hacía entrar a Octavio en la lucha por el poder en Roma. Al tratarse de un joven con pocos apoyos, ya que había sido adoptado recientemente, no parecía ser un oponente relevante. A los antiguos soldados de César se les puede atribuir lealtad o, tal vez, la idea de que una vez muerto su jefe caían en desgracia si no encontraban un sucesor.

El lector aún puede hacer otras inferencias de distinto tipo, por ejemplo prever que Octavio acabará convirtiéndose en el hombre más poderoso de Roma y que se vengará de aquellos que no le mostraron su apoyo, o pensar que los antiguos soldados de César se habrían dispersado por distintas regiones tras la muerte de su jefe. Por último, no se entendería del todo este texto si el lector no se percata de que se trata de un texto promocional cuya intención no es dar datos sobre la vida de Octavio, sino crear interés por la lectura y la adquisición de ese libro, titulado "Augusto".

En este contexto parece importante que se disponga de una taxonomía o clasificación de las inferencias orientada al aprendizaje y la práctica de la construcción de inferencias pragmáticas. Sin esa guía, los esfuerzos de mejora pueden ser ineficientes, ya que podría suceder que la práctica y la evaluación del aprendizaje de los alumnos se centrase sólo en algunos tipos de inferencias, dejando de lado otros.

En realidad, existen bastantes propuestas para la clasificación de las inferencias. Sin embargo, la situación es que estas propuestas utilizan, a veces, los mismos términos 
de una forma incompatible, no suelen estar concebidas para ayudar a la mejora de las habilidades inferenciales y ninguna de ellas es comúnmente aceptada. El lector interesado puede consultar León y Pérez (2003) para conocer los distintos criterios y sistemas de clasificación que se han utilizado.

Existen tres formas de clasificación que se encuentran con bastante frecuencia (Kispal, 2008). La primera es la distinción entre inferencias para mantener la cohesión del texto e inferencias elaborativas. La segunda, la distinción entre inferencias locales, que aseguran la coherencia en la comprensión de frases y párrafos, e inferencias globales. Y, en tercer lugar, se suele diferenciar entre inferencias que se realizan durante la lectura e inferencias que se realizan tras la lectura.

Chikalanga (1992) realizó un intento de proponer una clasificación de las inferencias que pudiera ser útil a los profesores de Lengua. Esta clasificación se basaba en la función de las inferencias, es decir, en su contribución a la comprensión del texto, y distingue tres grandes categorías de inferencias. La primera categoría son las inferencias léxicas, en las que se infiere el referente de los pronombres, las palabras desconocidas y las expresiones ambiguas.

La segunda categoría son las inferencias lógicas proposicionales, que se derivan lógicamente del contenido semántico del texto, es decir, están implicadas en el texto. Dentro de esta categoría se distinguen dos tipos: inferencias informativas y explicativas. Las informativas tratan sobre las circunstancias, y dan información sobre el qué, quién, cuándo y dónde. En cambio, las inferencias explicativas tratan sobre las motivaciones de los personajes, los condicionantes, las causas y las consecuencias de las acciones, dando información sobre el por qué y el cómo.

Chikalanga (1992) llamó inferencias pragmáticas a su tercera categoría, y las dividió en informativas, explicativas y evaluativas. Los dos primeros tipos coinciden con los tipos de inferencias proposicionales, con la diferencia de que las proposicionales se basarían en la información del texto, y las pragmáticas en los conocimientos del lector.

Otra clasificación con intención didáctica es la de Monfort y Monfort (2013), más centrada en el tipo de conocimiento que se necesita para construir las inferencias. La propuesta distingue cuatro tipos de inferencias. El primer tipo son las lógicas, en las que se precisan conocimientos básicos acerca de las relaciones causa-efecto y del significado de las palabras. El segundo tipo son las inferencias lógico-culturales, que necesitan conocimientos adquiridos en la transmisión cultural. El tercero son las inferencias lingüística,s que precisan del conocimiento de las reglas lingüísticas. Por último, el cuarto grupo son las inferencias pragmáticas, que precisan conocimientos de tipo mentalista, es decir, sobre los objetivos y las intenciones del emisor.

ISL, vol. 4, 2015, págs. 107-122 ISNN: 2340-8685
Ripoll Salcedo, J. C. (2015): Una clasificación de las inferencias pragmáticas orientada a la didáctica, Investigaciones Sobre Lectura, 4, 107-122. 
Puede producir extrañeza que la división que hacen estas clasificaciones entre inferencias lógicas y pragmáticas no coincida con la que se ha propuesto al principio del artículo, incluso que al hablar de inferencias pragmáticas se estén refiriendo a fenómenos distintos. Como se ha comentado, el uso de los mismos términos para referirse a conceptos diferentes es un problema bastante común en las taxonomías de inferencias.

\section{Una propuesta de clasificación}

Lo que se pretende aquí es proponer una clasificación de las inferencias compatible con las taxonomías existentes y que pueda ser comprensible y útil al profesorado para evaluar o desarrollar la habilidad inferencial de niños o adolescentes.

Dos decisiones importantes al plantear la clasificación han sido nombrar a los tipos de inferencia con un nombre genérico y utilizar como criterio de clasificación las preguntas que originarían los distintos tipos de inferencias.

Ya se ha expuesto y se ha comprobado cómo las clasificaciones que se han realizado hasta el momento han utilizado nombres como inferencias lógicas $\mathrm{o}$ inferencias pragmáticas para denominar a conceptos distintos. Esto también sucede con otros nombres, como inferencias elaborativas o conectivas. Para evitar perpetuar esa confusión la clasificación que se va a proponer utiliza una denominación neutra: inferencias de tipo I, II, III, IV y V.

Se ha utilizado como criterio de clasificación la pregunta o preguntas que originarían un tipo de inferencia, teniendo en cuenta que cuando se genera una inferencia se forma o se añade información que no estaba explícita en el texto, de modo que, si las inferencias añaden una información, siempre es posible preguntar por ella. Aquí es preciso señalar que hay autores, como Kintsch (1993) o León (2003), que consideran que las inferencias también pueden servir para reducir la información del texto, pero esta no es la perspectiva que se va a adoptar aquí.

Dentro de cada tipo se describirán, siempre que sea posible, las preguntas que originan las inferencias, las funciones que desempeñan o las diferentes clases que se pueden distinguir en ese tipo de inferencia, su relación con otras taxonomías de inferencias, especialmente con las dos que se han expuesto más detalladamente (Chikalanga, 1992; Monfort y Monfort, 2013) y los conocimientos que son necesarios para construirlas.

\subsection{Inferencias de tipo I}

Responden a preguntas como “¿a qué (o a quién) se refiere?”, “¿de qué (de quién) habla el texto cuando dice...?". Volviendo al texto sobre Octavio, encontramos varias expresiones referenciales, como "en ese momento", "el primer hombre de Roma", "su legítimo heredero" o "le tomó en serio", además de acciones con un sujeto sobrentendido o que ha aparecido con bastante anterioridad, como "formó un ejército". 
Las inferencias de tipo I parecen realizar, por lo menos, tres tipos de funciones:

1. Dar cohesión al texto relacionando referencias y referentes, por ejemplo, estableciendo que al decir "el primer hombre de Roma" se está hablando de Julio César y no del legendario fundador de la ciudad, Rómulo.

2. Solucionar ambigüedades. El lector tendrá que decidir si "ese momento" se refiere a los idus de marzo o al asesinato de Julio César. También hay ambigüedades producidas por la distancia entre el referente y la referencia. Por ejemplo, "formó un ejército" no tiene un sujeto, y el nombre más cercano es "Julio César", lo que podría llevar a algunos alumnos a pensar que fue él quien formo ese ejército.

3. Aventurar el significado de palabras y expresiones desconocidas. Un lector que desconozca lo que son los "idus de marzo" o un "magnicido" podría tratar de aventurar cuál es el significado de esas expresiones a partir de la información que encuentre en el texto y de sus conocimientos.

Para construir estas inferencias, el lector necesita conocimientos sintácticos, como el uso de los pronombres o las funciones de los términos de la oración y léxicos, como el significado de las palabras o palabras sinónimas.

Contrastando con las clasificaciones expuestas, las inferencias de tipo I coinciden con las inferencias léxicas de Chikalanga (1992) y con las inferencias lingüísticas de Monfort y Monfort (2013). Respecto a otras clasificaciones, se relacionan con las de tipo automático, obligatorias, perceptivas, basadas en el texto, referenciales o con las necesarias para la coherencia.

\subsection{Inferencias de tipo II}

Responden a preguntas como “¿por qué?” o “¿qué relación hay entre... y...?”. En este caso, su función es dar coherencia al texto estableciendo relaciones causa-efecto que no están explícitas en él. Si alguien recoge los pedazos de la jarra que se cayó es fácil inferir que el impacto de la caída hizo que la jarra se rompiera, pero no todas las inferencias de tipo II tienen que ver con relaciones causa-efecto físicas. Muchas de las inferencias de tipo II que se realizan en textos narrativos tienen que ver con los pensamientos y sentimientos de los personajes, especialmente con sus objetivos. Las aspiraciones y deseos de los personajes, así como sentimientos (envidia, venganza, altruismo, indignación) pueden ser la causa de sus acciones. En el texto que está sirviendo de ejemplo, los ciudadanos romanos no toman en serio a Octavio cuando se proclama sucesor de César, e inferimos que eso se debe a que, como era un joven recién adoptado, carecía de apoyos y de influencia. Son los antiguos soldados de su padre los que sí le apoyan, quizá por lealtad o por la perspectiva de poder volver a ganar influencia.

Una información que puede pasar desapercibida es que Octavio "formó un ejército". Una actuación tan extraña no llama la atención porque la relacionamos con su

ISL, vol. 4, 2015, págs. 107-122 ISNN: 2340-8685
Ripoll Salcedo, J. C. (2015): Una clasificación de las inferencias pragmáticas orientada a la didáctica, Investigaciones Sobre Lectura, 4, 107-122. 
objetivo de convertirse en el sucesor de Julio César, es decir, en el hombre más poderoso de Roma, y disponer de un ejército es una fuente de poder.

Para la construcción de estas inferencias el lector necesita diversos tipos de conocimientos: conocimientos sobre las relaciones causa-efecto básicas y el funcionamiento de las cosas, conocimientos sobre el pensamiento y el comportamiento de las personas y conocimientos sobre el tema del texto.

Las inferencias de tipo II tienen mucha relación con las inferencias explicativas de Chikalanga (1992), tanto con las lógicas-explicativas como con las pragmáticasexplicativas. En la clasificación de Monfort y Monfort (2013) podrían ser parte de las inferencias lógicas, lógico-culturales y pragmáticas. En otras clasificaciones, se superpondrían parcialmente con las inferencias puente, hacia atrás, automáticas, obligatorias, necesarias para la coherencia, basadas en los conocimientos del lector o de meta.

\subsection{Inferencias de tipo III}

En este caso las preguntas son del tipo “¿qué sucederá?”, “¿qué se puede predecir sabiendo que...?” o “¿para qué?”. Tras leer el texto que está sirviendo de ejemplo, hay lectores que pueden pensar que habrá algún tipo de confrontación entre Octavio y los otros dos hombres más poderosos de Roma. Otros lectores pueden predecir que Octavio se aliará con alguno de ellos.

La función principal de las inferencias de tipo III es hacer hipótesis sobre los sucesos del texto, por ejemplo, las consecuencias de lo que narra o describe el texto en el mundo físico o en los estados mentales de los personajes. Estas predicciones no son necesarias para comprender el texto. Incluso muchas veces sucede que la información que se proporciona posteriormente indica que no eran correctas. Aunque es un tema muy poco estudiado, este tipo de inferencias podría relacionarse con el interés que despierta el texto (Campion, Martins y Wilhelm, 2009).

Las clasificaciones de Chikalanga (1992) y Monfort y Monfort (2013) no distinguen una clase que englobe las inferencias de tipo predictivo. Las inferencias de tipo III formarían parte de las inferencias pragmáticas-explicativas, lógicas, lógicoculturales y pragmáticas. Se trata, casi, de las mismas categorías que se han mencionado al hablar de las inferencias de tipo II. Hay que tener en cuenta que tanto las inferencias de tipo II como las de tipo III tienen un componente causal, de modo que esta coincidencia no es extraña.

Sin embargo, en la clasificación que se está proponiendo se han separado, en lugar de construir una categoría de inferencias causales que englobase las de tipo II y las de tipo III. Una razón para esta división es que ambos tipos de inferencia responden a 
distintos tipos de preguntas, el tipo II a "¿qué relación hay entre...?”, y el tipo III a “¿qué se puede predecir sabiendo qué?”. Eso hace que haya diferencias en la forma como se construyen y en su función.

Las inferencias de tipo II relacionan causalmente dos elementos que aparecen en el texto de modo que se pueda formar una representación coherente de su significado. En cambio, en las inferencias de tipo III no se establecen relaciones entre elementos del texto, sino que se infieren nuevos elementos. La información de que Octavio "formó un ejército" la podemos relacionar con la idea de que Octavio se proclamó sucesor de César, aportando la información de que esa sucesión implica una lucha para hacerse con el poder de Roma. Eso sería una inferencia de tipo II. En cambio una inferencia de tipo III aporta un nuevo elemento, por ejemplo, batallas (de las que no habla el texto) contra los partidarios de Marco Antonio y de Lépido.

En cuanto a su función, las inferencias de tipo II son explicativas y garantizan la cohesión del texto, estableciendo relaciones entre sus elementos, mientras que las inferencias de tipo III no se establecen relaciones entre elementos del texto, sino que anticipan nuevos elementos. Por eso, en otras taxonomías se suele considerar que las inferencias de tipo II son necesarias (dan cohesión al texto) y hacia atrás (relacionan un elemento del texto con otro que ya ha aparecido), mientras que las inferencias de tipo III son hacia adelante (anticipan nuevos elementos).

Al tener un carácter causal, los conocimientos que se necesitan para hacer inferencias de tipo III son muy parecidos a los que se precisan para el tipo II: conocimientos generales y sobre el tema del texto. Pero una característica de las inferencias de tipo III es que pueden ser infinitas, como se comentará al hablar de las de tipo IV, que también pueden serlo. Sabemos que los lectores sólo suelen realizar unas pocas predicciones entre la infinidad que sería posible, y probablemente el conocimiento sobre los géneros literarios y sus convenciones ayuda a delimitar qué predicciones son razonables. En el caso del texto de Octavio, damos por supuesto que el autor nos dará informaciones que sean sostenibles con datos históricos y podemos descartar de antemano que un genio conceda deseos al protagonista o que un animal le dé consejos.

\subsection{Inferencias de tipo IV}

Este tipo de inferencias es el más vago de todos y responde a preguntas como “¿cuándo?”, “¿dónde?”, “cómo?”, “¿con qué?”, “¿de qué color?” y muchas otras de ese tipo. Se podrían englobar en una pregunta general que sería "¿qué más se puede decir sobre esto?". Podemos imaginar cómo iba vestido Octavio, pensar si el asesinato de Julio César fue por la mañana o por la tarde o si la estatua de Pompeyo lo representaba con los brazos cruzados.

ISL, vol. 4, 2015, págs. 107-122 ISNN: 2340-8685
Ripoll Salcedo, J. C. (2015): Una clasificación de las inferencias pragmáticas orientada a la didáctica, Investigaciones Sobre Lectura, 4, 107-122. 
Los conocimientos generales y los conocimientos concretos sobre el tema del texto pueden suscitar infinidad de inferencias de este tipo. Estas inferencias serán más creibles si el lector también cuenta con conocimientos sobre el género literario del texto y sobre el autor y su contexto. En este caso, si un historiador nos está hablando de un asesinato ocurrido unos años antes de nuestra era, no sería adecuado pensar que el arma homicida fue una pistola.

En la clasificación de Chikalanga (1992) las inferencias de tipo IV se corresponden con las inferencias informativas, tanto lógicas como pragmáticas, y en la de Monfort y Monfort (2013) serían una parte de las inferencias lógico-culturales. En otras clasificaciones se les llama inferencias elaborativas y, a veces, se distinguen algunos tipos particulares que reciben nombres como las inferencias de instrumento o las emocionales.

Tal como se ha visto, en un texto se podrían construir infinidad de inferencias de tipo IV, algo que también sucede con las de tipo III. La mayoría de las que se podrían construir resultarían irrelevantes. Por ejemplo, el significado del texto apenas cambia si pensamos que la estatua de Pompeyo era de mármol o si pensamos que era de granito. Afortunadamente, durante la lectura no se suelen realizar inferencias de tipo IV a no ser que el texto las favorezca (Harmon-Vukic, Guéraud, Lassonde y O'Brien, 2009) o los objetivos del lector faciliten su formación (van Meter y Pressley, 1994; Allbritton, 2004).

\subsection{Inferencias de tipo $\mathrm{V}$}

Las inferencias de tipo V responden a preguntas como "¿qué me están contando aquí?" o "¿qué quiere decir todo esto?". No se trata de preguntas relacionadas con un elemento concreto del texto, como una palabra o una estructura desconocida, sino de preguntas que consideran el texto en su conjunto o una parte amplia de él. La utilidad de este tipo de inferencias es evidente cuando se deben interpretar ciertas formas de lenguaje figurado como las alegorías, la ironía o metáforas novedosas, o cuando se encuentra una moraleja o enseñanza en un texto. En cierta forma, las inferencias de tipo V plantean el tema del texto cuando no está explícito.

Probablemente son el tipo de inferencia menos frecuente, para empezar, porque no son pertinentes en todos los textos. En el texto sobre Octavio que está sirviendo de ejemplo, un lector competente idendificará que su propósito es promocionar un libro, titulado "Augusto". Pero en muchas ocasiones la intención del autor es transparente, por ejemplo, describe las partes de un volcán porque quiere explicar cuáles son las partes de un volcán.

Lógicamente, para formar estas inferencias, es necesario contar con conocimientos sobre el mundo y sobre el tema del texto, pero también es útil tener 
conocimientos sobre el autor, su contexto y sus intenciones y sobre los géneros literarios y sus convenciones.

En la clasificación de Chikalanga (1992) no aparece ninguna categoría que coincida con este tipo de inferencias, y en la de de Monfort y Monfort (2013) pueden coincidir, en parte, con las inferencias pragmáticas. Entre los tipos de inferencia propuestos en otras taxonomías, las inferencias de tipo $\mathrm{V}$ estarían muy relacionadas con las inferencias globales y las temáticas. No obstante, en algunos modelos de comprensión se considera que el tipo de operaciones que se están describiendo no forman parte de la comprensión inferencial, sino que son propios de otro nivel que sería la comprensión crítica o evaluativa (Basaraba, Yovanoff, Alonzo y Tindal, 2012). Aunque habría operaciones que serían propias de la comprensión crítica, como estar de acuerdo con el contenido del texto o no, o las reacciones emocionales producidas por su lectura, las inferencias de tipo $\mathrm{V}$, tal como se han descrito aquí aportan información para formar una representación mental del texto combinando la información que aparece en el propio texto con los conocimientos del lector, por lo tanto, podrían considerarse como auténticas inferencias.

\section{Algunos datos sobre el uso de estas inferencias en la edad escolar}

Aunque no sea suficiente para validar esta clasificación, un buen punto de partida es mostrar cómo los alumnos realizan los tipos de inferencias que se han propuesto. Sin ánimo de ser exhaustivo, se dan aquí las referencias a algunos estudios que aportan datos sobre la existencia de estos tipos de inferencias en alumnado de educación infantil, primaria o secundaria.

Antes de considerar las inferencias pragmáticas, es interesante citar los trabajos de Oakhill (1982) y Cain, Oakhill y Bryant (2004), en los que la mayor parte de las inferencias que se exploraban eran del tipo lógico. Por ejemplo, si un avión vuela por encima de una casa y esa casa está en Asturias, los alumnos pueden inferir que el avión está volando por encima de Asturias.

Respecto a las inferencias de tipo I sabemos que los alumnos tienen la habilidad para encontrar el referente de pronombres (Bowyer-Crane y Snowling, 2005; DuqueAristizábal y Vera-Márquez, 2010), para identificar un elemento del texto, por ejemplo "el zumo de naranja" al que se hace referencia con otra expresión, como "la bebida" (Cain y Oakhill, 1999; Villalonga, Padilla y Burin, 2014) y que son capaces de inferir el significado de palabras desconocidas a partir de la información que ofrece el texto (Cain, Oakhill y Lemmon, 2004).

También se han recogido datos sobre cómo los alumnos relacionan causalmente (inferencias de tipo II) elementos del texto como el inicio de un incendio y coger un cubo de agua (Cain, Oakhill, Barnes y Bryant, 2001; Laing y Kamhi, 2002; Bowyer-

ISL, vol. 4, 2015, págs. 107-122 ISNN: 2340-8685
Ripoll Salcedo, J. C. (2015): Una clasificación de las inferencias pragmáticas orientada a la didáctica, Investigaciones Sobre Lectura, 4, 107-122. 
Crane y Snowling, 2005; Lynch y van den Broek, 2007; Bowyer-Crane y Snowling, 2010; Duque-Aristizábal y Vera-Márquez, 2010).

Aunque se trate de una información obvia, los alumnos en edad escolar también son capaces de hacer inferencias de tipo III, es decir, predicciones (Laing y Kamhi, 2002; Bowyer-Crane y Snowling, 2005; Duque-Aristizábal y Vera-Márquez, 2010).

Las inferencias de tipo IV tienen muchas formas posibles. Tenemos datos que indican que los alumnos las realizan (Bowyer-Crane y Snowling, 2010) y son capaces de inferir el lugar donde transcurren los sucesos del texto (Cain y Oakhill, 1999; Villalonga et al. 2014), los sentimientos o emociones de los personajes de una narración (Bowyer-Crane y Snowling, 2005; Duque-Aristizábal y Vera-Márquez, 2010), el color de un elemento (Cain et al., 2001) o el instrumento que se emplea para una acción (Cain y Oakhill, 1999).

Las inferencias de tipo $\mathrm{V}$ han sido, generalmente, menos estudiadas, pero los niños son capaces de inferir el significado adecuado de expresiones irónicas (Banasik, 2013) o la moraleja de un relato (Duque-Aristizábal y Vera-Márquez, 2010; Narvaez, Gleason y Mitchell, 2010).

No todos los alumnos realizan siempre estos tipos de inferencias. Incluso parece haber una relación entre su construcción y el nivel de comprensión de los alumnos que se ha identificado en numerosos estudios (Cain y Oakhill, 1999; Cain et al. 2001; Laing y Kamhi, 2002; Cain et al. 2004; Bowyer-Crane y Snowling, 2005). Esta relación entre habilidad para construir inferencias y comprensión no parece encontrarse cuando se consideran las inferencias de tipo III (Laing y Kamhi, 2002).

Por último, existen algunas investigaciones en las que se han estudiado los efectos de programas para mejorar la habilidad de construcción de inferencias de los alumnos. En estas investigaciones se han encontrado mejoras en la habilidad inferencial o en la comprensión tras entrenar la realización de inferencias de tipo II (McMaster, van den Broek, Espin et al., 2012), tipo III y tipo IV (Yuill y Oakhill, 1988).

\section{Discusión}

Se ha presentado un clasificación con cinco tipos de inferencias que se realizan en algún momento del proceso de comprensión lectora (o del lenguaje oral). Como se ha visto a lo largo de la exposición, los cinco tipos tienen coherencia con las clases de inferencia que se proponen en otras clasificaciones, aunque las agrupan de otra manera. La razón por la que la agrupación es distinta es porque el criterio empleado para establecer las distintas clases de inferencias es diferente a los empleados anteriormente. Para distinguir clases de inferencias se han utilizado criterios como el momento en el que se realizan, el tiempo que tardan en formularse, los recursos cognitivos que 
necesitan o su dirección (León y Pérez, 2003). Las clasificaciones más orientadas a la didáctica o a la intervención para mejorar la comprensión han seguido criterios como la función de las inferencias (Chikalanga, 1992) o el tipo de conocimientos que se necesitan para construirlas (Monfort y Monfort, 2013). En este caso se ha utilizado como criterio el tipo de información que aportan esas inferencias al lector.

Tal como se sugería en la introducción, contar con una clasificación de los distintos tipos de inferencia es una ayuda para realizar una evaluación y seguimiento adecuado del desarrollo de las habilidades de comprensión del alumnado, sin dejar de lado ningún aspecto y es también una ayuda para la programación de actividades que conduzcan a la mejora de esas habilidades de comprensión. En este caso, además, como cada clase de inferencia está vinculada a un tipo de preguntas, la aplicación es mucho más fácil, porque los profesores podrían trabajar la comprensión inferencial de forma bastante extensa realizando las preguntas que se proponen, aun sin conocer las bases de la clasificación.

Conviene recordar que lo que se encuentra aquí es una mera propuesta y su mayor limitación es que apenas está respaldada por datos empíricos. Aunque existen datos que indican que todos los tipos de inferencia que se han propuesto son realizados por el alumnado de educación infantil, primaria y secundaria, hay muy poca información sobre cómo es su evolución.

A modo de conclusión se podría decir los docentes y las personas interesadas en el desarrollo de la comprensión cuentan con una sencilla guía orientada a la práctica para poder trabajar la comprensión inferencial de forma extensa.

\section{Bibliografía}

Abusamra, V., Ferreres, A., Raiter, A., de Beni R. y Cornoldi, C. (2010). Test leer para comprender. Buenos Aires: Paidós.

Allbritton, D. (2004). Strategic production of predictive inferences during comprehension. Discourse Processes, 38(3), 309-322.

Banasik, N. (2013). Non-literal speech comprehension in preschool children - An example from a study on verbal irony. Pyshcology of Language and Communication, 17(3), 309-323.

Basaraba, D., Yovanoff, P., Alonzo, J. y Tindal, G. (2012). Examining the structure of reading comprehension: do literal, inferential, and evaluative comprehension truly exist? Reading and Writing, 26, 349-379.

ISL, vol. 4, 2015, págs. 107-122 ISNN: 2340-8685
Ripoll Salcedo, J. C. (2015): Una clasificación de las inferencias pragmáticas orientada a la didáctica, Investigaciones Sobre Lectura, 4, 107-122. 
Bowyer-Crane, C. y Snowling, M. (2005). Assesing children's inference generation: what do tests of reading comprehension measure? British Journal of Educational Psychology, 75, 189-201.

Bowyer-Crane, C. y Snowling, M. J. (2010). Turning frogs into princes: can children make inferences from fairy tales? Reading and Writing, 23, 19-29.

Cain, K. y Oakhill, J. V. (1999). Inference making ability and its relation to comprehension failure

in young children. Reading and Writing: An Interdisciplinary Journal, 11, 489-503.

Cain, K. Oakhill, J. V., Barnes, M. A. y Bryant, P. E. (2001). Comprehension skill, inference-making ability, and their relation to knowledge. Memory \& Cognition, 29(6), 850-859. Recuperado el 3 de marzo, 2015 de https://www.researchgate.net/profile/Jane Oakhill/publication/11639427 Compr ehension skill inferencemaking_ability_and their_relation_to_knowledge/links/0046351deabfbb852600 $\underline{0000 . p d f}$

Cain, K. Oakhill, J. V. y Bryant, P. (2004). Children's reading comprehension ability: concurrent prediction by working memory, verbal ability, and component skills. Journal of Educational Psychology, 96(1), 33-42.

Cain, K. Oakhill, J. V. y Lemmon, K. (2004). Individual differences in the inference of word meanings from context: the influence of reading comprehension, vocabulary knowledge, and memory capacity. Journal of Educational Psychology, 96(4), 671-681.

Campion, N., Martins, D. y Wilhelm, A. (2009). Contradictions and predictions: Two sources of uncertainty that raise the cognitive interest of readers. Discourse Processes, 46(4), 341-368.

Chikalanga, I. (1992). A suggested taxonomy of inferences for the reading teacher. Reading in a Foreign Language, 8(2), 697-709. Recuperado el 3 de marzo, 2015 de http://www.nflrc.hawaii.edu/rfl/PastIssues/rfl82chikalanga.pdf 
Cuetos, F., Rodríguez, B., Ruano, E., y Arribas, D. (2007). Prolec-R. Batería de evaluación de los procesos lectores revisada. Madrid: TEA.

Duque-Aristizábal, C. P., y Vera-Márquez, A. V. (2010). Exploración de la comprensión inferencial de textos narrativos en niños de preescolar. Revista Colombiana de Psicología, 19(1), 21-35. Recuperado el 3 de marzo, 2015 de http://dialnet.unirioja.es/descarga/articulo/3641261.pdf

Fernández, M. d. O. (2008). Lapiceros. Lengua 1. Madrid: Bruño.

Harmon-Vukic, M., Guéraud, S., Lassonde, K. A. y O'Brien, E. J. (2009). The activation and instantation of instrumental inferences. Discourse Processes, 46, 467-490.

Kintsch, W. (1993). Information accretion and reduction in text processing: inferences. Discourse Processes, 16, 193-202.

Kispal, A. (2008). Effective teaching of inference skills for reading. Literature review. Berkshire: National Foundation for Educational Research. Recuperado el 3 de marzo, 2015 de http://decd.sa.gov.au/northernadelaide/files/links/inference paper.pdf

Laing, S. P. y Kamhi, A. G. (2002). The use of think-aloud protocols to compare inferencing abilities in average and below-average readers. Journal of Learning Disabilities, 35(5), 436-447.

León, J. A. (2003). Una introducción a los procesos de inferencias en la comprensión del discurso escrito. En J. A. León (Ed.). Conocimiento y discurso. Claves para inferir y comprender (pp. 23-43). Madrid: Pirámide.

León, J. A. y Pérez, O. (2003). Taxonomías y tipos de inferencias. En J. A. León (Ed.). Conocimiento y discurso. Claves para inferir y comprender (pp. 45-68). Madrid: Pirámide.

Lynch, J. S. y van de Broek, P. (2007). Understanding the glue of narrative structure: Children's on- and of-line inferences about characters' goals. Cognitive Development, 22, 323-340.

Ripoll Salcedo, J. C. (2015): Una clasificación de las inferencias pragmáticas orientada a la didáctica, Investigaciones Sobre Lectura, 4, 107-122. 
McMaster, K. L., van den Broek, P., Espin, C. A., White, M. J., Rapp, D. N., Kendeou, P., Bohn-Getler, C. M. y Carlson, S. (2012). Make de right connections: differential effects of reading intervention for subgroups of comprehenders. Learning and Individual Differences, 22, 100-111.

Monfort, I. y Monfort, M. (2013). Inferencias y comprensión verbal en niños con trastornos del desarrollo del lenguaje. Revista de Neurología, 56(Supl 1), S141S146. Recuperado el 3 de marzo, 2015 de http://www.neurologia.com/pdf/Web/56S01/bjS01S141.pdf

Narvaez, D., Gleason, T. y Mitchell, C. (2010). Moral virtue and practical wisdom: theme comprehension in children, youth and adults. Journal of Genetic Psychology, 171(4), 363-388. Recuperado el 3 de marzo, 2015 de http://www3.nd.edu/ dnarvaez/documents/NarvaezGleasonGenPsy2010.pdf

Oakhill, J. (1982). Constructive processes in skilled and less skilled comprehenders' memory for sentences. British Journal of Psychology, 73(1), 13-20.

Real Decreto 126/2014, de 28 de febrero, por el que se establece el currículo básico de la Educación Primaria. Recuperado el 3 de marzo, 2015 de http://www.boe.es/diario boe/txt.php?id=BOE-A-2014-2222

Ripoll, J. C. y Aguado, G. (2014). La mejora de la comprensión lectora en español: un meta-análisis. Revista de Psicodidáctica, 19(1), 27-44. Recuperado el 3 de marzo, 2015 de http://www.ehu.eus/ojs/index.php/psicodidactica/article/download/9001/9935

Van Metter, P. y Pressley, M. (1994). Encoding of instruments when 10- to 14- year-olds process isolated instrument-implicit sentences: More evidence of improved encoding during childhood resulting from elaborative instructions. Journal of Educational Pyshcology, 86(3), 402-412.

Villalonga, M. M., Padilla, C. y Burin, D. (2014). Relaciones entre descodificación, conocimiento léxico-semántico e inferencias en niños de escolaridad primaria. Interdisciplinaria, 31(2), 259-274. Recuperado el 3 de marzo, 2015 de http://www.scielo.org.ar/scielo.php?pid=S1668- 


\section{$\underline{70272014000200005 \& \text { script }=\text { sci arttext }}$}

Yuill, N. y Oakhill, J. (1988). Effects of inference awareness training on poor reading comprehension. Applied Cognitive Psychology, 2(1), 33-45.

ISL, vol. 4, 2015, págs. 107-122 ISNN: 2340-8685
Ripoll Salcedo, J. C. (2015): Una clasificación de las inferencias pragmáticas orientada a la didáctica, Investigaciones Sobre Lectura, 4, 107-122. 